\title{
Minimal predictors in hat problems
}

\author{
by \\ Christopher S. Hardin and Alan D. Taylor (Schenectady, NY)
}

\begin{abstract}
We consider a combinatorial problem related to guessing the values of a function at various points based on its values at certain other points, often presented by way of a hat-problem metaphor: there are a number of players who will have colored hats placed on their heads, and they wish to guess the colors of their own hats. A visibility relation specifies who can see which hats. This paper focuses on the existence of minimal predictors: strategies guaranteeing at least one player guesses correctly, regardless of how the hats are colored. We first present some general results, in particular showing that transitive visibility relations admit a minimal predictor exactly when they contain an infinite chain, regardless of the number of colors. In the more interesting nontransitive case, we focus on a particular nontransitive relation on $\omega$ that is elementary, yet reveals unexpected phenomena not seen in the transitive case. For this relation, minimal predictors always exist for two colors but never for $\aleph_{2}$ colors. For $\aleph_{0}$ colors, the existence of minimal predictors is independent of ZFC plus a fixed value of the continuum, and turns out to be closely related to certain cardinal invariants involving meager sets of reals.
\end{abstract}

1. Introduction. Our set-theoretic notation is fairly standard. If $X$ and $Y$ are sets, then ${ }^{X} Y$ denotes the set of all functions from $X$ to $Y$, and $X-Y$ is the set-theoretic difference of $X$ and $Y$. We use $[X]^{k}$ to denote the set of all $k$-element subsets of $X$, and $[X]^{\omega}$ for the set of all countably infinite subsets of $X$. If $f$ is a function and $A$ is a subset of the domain of $f$, then $f \mid A$ is the restriction of $f$ to the set $A$.

In very general terms, the class of problems we are interested in ask how successfully one can predict an arbitrary function's value at a given point based on information about its values elsewhere. We explored this in the topological context in [HT09] and with respect to functions of time in HT08b. In the present paper, however, we rely heavily on the hat-problem metaphor described in [HT08a], the generic version of which goes as follows. A group of players is to have hats of various colors placed on their heads.

2010 Mathematics Subject Classification: Primary 03E05; Secondary 03E17.

Key words and phrases: meager sets, Martin's axiom, dependent choice, continuum hypothesis. 
Each player can see the hats of some of the other players, but not his or her own. Except for a strategy session before the hats are placed, there is no communication. The players are required to simultaneously submit a guess of their own hat color.

For our purposes here, the specification of who can see whom will be given by a directed graph $V$ with no loops, called a visibility graph (or visibility relation), with an edge from $p$ to $q$ indicating that $p$ can see (the hat worn by) $q$. We let $V(p)=\{q: p V q\}$, so $V(p)$ is the set of players that are visible to $p$. The set of colors will be a finite or infinite cardinal $\nu$, and a hat assignment is an element of ${ }^{P} \nu$.

A strategy for a player $p \in P$ is a function $S_{p}:{ }^{P} \nu \rightarrow \nu$ with the property that if $h$ and $g$ are hat assignments with $h|V(p)=g| V(p)$, then $S_{p}(h)=S_{p}(g)$. Thus, a strategy for player $p$ provides him with a guess for his hat color given a hat assignment $h$, and this guess depends only on the hats that he can see. Notice that this guess is correct iff $S_{p}(h)=h(p)$. A predictor $S$ is a sequence $\left\langle S_{p}: p \in P\right\rangle$ where $S_{p}$ is a strategy for player $p$. That said, we will often regard a predictor as a function $S:{ }^{P} \nu \rightarrow{ }^{P} \nu$, with $S(h)(p)=S_{p}(h)$.

Additionally, if we have a collection $\mathcal{C}$ of subsets of $P$ then the notation

$$
\langle P, V\rangle \rightarrow\langle\mathcal{C}\rangle_{\nu}
$$

denotes the assertion that if $P$ is the set of players and $V$ is the visibility graph, then there exists a predictor ensuring that, for every hat assignment with $\nu$ colors, the set of players who correctly guess their own hat color is in the collection $\mathcal{C}$. In specifying $\mathcal{C}$, we use a cardinal $\kappa$ itself to denote the collection of subsets of $P$ of cardinality at least $\kappa$, so 1 denotes the collection of nonempty subsets of $P$, and $\omega$ denotes the collection of infinite subsets of $P$. For visibility, we use Full to denote the complete directed graph with no loops; this corresponds to full visibility subject to the constraint that no one sees his or her own hat.

Our interest here is in the case where $P$ is infinite. Early precursors to such considerations date back at least to Fred Galvin's work Gal65] in the mid-1960s and his initial application to provide a simple proof of a theorem of Jerome Malitz dealing with a class of infinite-quantifier formulas introduced by Leon Henkin. Later, Galvin and Karel Prikry GP76] used this same result in their investigations of Jonsson algebras. Other results can be recast in terms of the hat-problem metaphor, including the work of Andreas Blass Bla94 on the so-called Specta-Eda number: the size of the smallest subgroup $G$ of $\mathbb{Z}^{\omega}$ containing all unit vectors which still has the property that every homomorphism $h: G \rightarrow \mathbb{Z}$ annihilates almost all unit vectors. These investigations by Blass were followed up in a series of papers by Jörg Brendle and Saharon Shelah [Bre95, BS96, Bre03, BS03. 
More recently, Yuval Gabay and Michael O'Connor observed that with full visibility there is a predictor guaranteeing at most finitely many errors (a finite-error predictor), regardless of the set of players or colors; see HT08a. In our notation, this says that for every set $P$ and every cardinal $\nu$, we have $\langle P$, Full $\rangle \rightarrow\langle\mathcal{F}\rangle_{\nu}$ where $\mathcal{F}$ denotes the filter of cofinite subsets of $P$. The predictor here is easy to describe. One defines an equivalence relation $\approx$ on ${ }^{P} \nu$ by $f \approx g$ if they only differ at finitely many points in $P$. By the axiom of choice, there is a set $C$ consisting of exactly one representative from each equivalence class. The players fix such a set $C$, and under a hat assignment $h$, each player guesses according to the unique $g \in C$ with $g \approx h$.

Rather than seeking finite-error predictors with full visibility, our interest here lies at an opposite extreme. Specifically, we seek minimal predictors: predictors that guarantee at least one correct guess. In sharp contrast to earlier results, it turns out that the number of colors now plays a remarkably prominent role. Fairly simple situations quickly lead to results independent of ZFC and even ZFC with a fixed value of the continuum.

The rest of this paper is organized as follows. In Section 2, we begin with the weakest theory of interest to us and exploit an infinite exponent partition relation to show that even $\langle\omega$, Full $\rangle \rightarrow\langle 1\rangle_{\omega}$ is not a theorem of ZF + DC. Because of this, we restrict all later considerations to ZFC. We also show that finite visibility (i.e., each player sees finitely many other players) is insufficient for the existence of minimal predictors, even for two colors, except in fairly trivial cases.

In Section 3, we show that ZFC is sufficient to provide a very satisfying answer to the question of when minimal predictors exist in the special case in which the visibility relation is transitive. Namely, for transitive relations, minimal predictors exist iff there is an infinite chain of visibility.

We turn next to nontransitive visibility relations and focus on a highly nontransitive relation on $\omega$ wherein evens see higher-numbered odds and odds see higher-numbered evens. We denote this directed graph by EO, for "even-odd". In Section 4 we show that, in ZFC, the relation $\langle\omega, \mathrm{EO}\rangle \rightarrow$ $\langle 1\rangle_{2}$ holds and the relation $\langle\omega, \mathrm{EO}\rangle \rightarrow\langle 1\rangle_{\omega_{2}}$ fails. We also show here that $\langle\omega, \mathrm{EO}\rangle \rightarrow\langle 1\rangle_{\omega_{1}}$ is a consequence of $\mathrm{CH}$. We do not know if $\langle\omega, \mathrm{EO}\rangle \rightarrow\langle 1\rangle_{3}$ is provable in ZFC or if $\langle\omega, \mathrm{EO}\rangle \rightarrow\langle 1\rangle_{\omega_{1}}$ implies $\mathrm{CH}$.

In Section 5 , we consider the theory ZFC $+2^{\aleph_{0}}=\aleph_{2}$ and prove that $\langle\omega, \mathrm{EO}\rangle \rightarrow\langle 1\rangle_{\omega}$ is independent of this theory. The positive result uses MA and the negative one the addition of $\aleph_{2}$ random reals and some surprising connections with the ideal of meager sets of reals. We conclude in Section 6 by collecting together a number of open questions.

Before continuing, we make the following observation, which we use twice. 
Proposition 1.1. If $P=\omega$ and $V$ is a subrelation of the ordering $<$, then $\langle\omega, V\rangle \rightarrow\langle 1\rangle_{\nu}$ iff $\langle\omega, V\rangle \rightarrow\langle\omega\rangle_{\nu}$.

Proof. The right-to-left implication is trivial. For the left-to-right implication, it suffices to show that any predictor failing to guarantee infinitely many correct guesses must actually fail to guarantee even one correct guess. Let $S$ be a predictor with a hat assignment $f$ under which the set $C=\{n \in \omega: S(f)(n)=f(n)\}$ of correct guesses is finite. If $C=\emptyset$, we are done. Otherwise, let $m=\max C$. We will define a hat assignment $f^{\prime}$ that makes everyone wrong, by starting at $m$ and working downward. Let $f^{\prime}(k)=f(k)$ for $k>m$. Then $S\left(f^{\prime}\right)(m)$ is well-defined, and we can define $f^{\prime}(m) \neq S\left(f^{\prime}\right)(m)$; then $S\left(f^{\prime}\right)(m-1)$ is well-defined, and we can define $f^{\prime}(m-1) \neq S\left(f^{\prime}\right)(m-1)$, and so on. Eventually, we have defined $f^{\prime}$ such that $\left\{n \in \omega: S\left(f^{\prime}\right)(n)=f^{\prime}(n)\right\}=\emptyset$.

2. The need for $\mathrm{AC}$ and infinite visibility. In the further parts of this paper, we seek combinations of set-theoretic axioms and visibility assumptions adequate to yield positive results for minimal predictors. But we begin with two negative results. The first asserts that ZF $+\mathrm{DC}$ is not a sufficiently strong theory to yield a minimal predictor on $\omega$ with infinitely many colors, even with full visibility. The second asserts that finite visibility only suffices in fairly trivial situations even with two colors.

It was shown in [HT08a] that one cannot obtain the Gabay-O'Connor theorem, even with two colors and $P$ countable, without some nontrivial version of the axiom of choice. On the other hand, with full visibility and finitely many colors, there are trivial predictors ensuring that infinitely many players guess correctly (e.g., with any set of players all of whom can see all but finitely many of the other players, we can number the colors and have each player guess that his hat color is the lowest-numbered color that he sees occurring infinitely many times).

To prove that $\mathrm{ZFC}+\mathrm{DC}$ is too weak to yield a minimal predictor with infinitely many colors and full visibility, we need first to recall that the notation $\omega \rightarrow(\omega)_{2}^{\omega}$ means that for every function $f:[\omega]^{\omega} \rightarrow 2$, there exists an infinite set $X$ such that $f$ is constant on $[X]^{\omega}$. Similarly, the notation $\omega \rightarrow[\omega]_{\omega}^{\omega}$ means that for every function $f:[\omega]^{\omega} \rightarrow \omega$, there exists an infinite set $X$ and a number $n \in \omega$ such that $n \notin f\left([X]^{\omega}\right)$. A. R. D. Mathias Mat77] showed that if ZFC plus the existence of a large cardinal is consistent, then so is ZF + DC together with the assertion that $\omega \rightarrow(\omega)_{2}^{\omega}$. We only need the (apparently) weaker assertion $\omega \rightarrow[\omega]_{\omega}^{\omega}$, and the following easy consequence of it.

Lemma 2.1. Assume $\omega \rightarrow[\omega]_{\omega}^{\omega}$. Then for every $X \in[\omega]^{\omega}$ and every function $f:[X]^{\omega} \rightarrow \omega$, there exists an infinite set $X^{\prime} \subseteq X$ and a number $n \in X$ such that $n<\min X^{\prime}$ and for every $Y \in\left[X^{\prime}\right]^{\omega}$, we have $f(Y) \neq n$. 
Proof. Given $f:[X]^{\omega} \rightarrow \omega$, define $g:[X]^{\omega} \rightarrow X$ by setting $g(Y)=f(Y)$ if $f(Y) \in X$ and $g(Y)=\min X$ otherwise. Because $\omega \rightarrow[\omega]_{\omega}^{\omega}$, we can (by identifying $X$ with $\omega)$ choose $X^{\prime \prime} \in[X]^{\omega}$ and $n \in X$ such that $n \notin g\left(\left[X^{\prime \prime}\right]^{\omega}\right)$. Letting $X^{\prime}=X^{\prime \prime}-\{0, \ldots, n\}$ works.

The following theorem is inspired by results in Galvin and Prikry's 1976 paper [GP76].

Theorem 2.2. (ZF + DC) If $\omega \rightarrow[\omega]_{\omega}^{\omega}$ holds, then $\langle\omega$, Full $\rangle \rightarrow\langle 1\rangle_{\omega}$ fails.

Proof. Assume that $\omega \rightarrow[\omega]_{\omega}^{\omega}$ and that $S=\left\langle S_{n}: n \in \omega\right\rangle$ is a predictor for full visibility. We will inductively construct a sequence $\left\langle\left(x_{n}, X_{n}\right): n \in \omega\right\rangle$ of pairs such that $x_{0}<x_{1}<\cdots$ and $X_{0} \supset X_{1} \supset \cdots$ and such that for each $n \in \omega$, the following hold:

(a) $x_{n} \in X_{n}$ and $X_{n} \in[\omega]^{\omega}$.

(b) $x_{n}<\min \left(X_{n+1}\right)$.

(c) For each $Y \in\left[X_{n+1}\right]^{\omega}$, if $\left\langle y_{0}, y_{1}, \ldots\right\rangle$ is the increasing enumeration of $Y$, then $S_{n}\left(\left\langle x_{0}, \ldots, x_{n-1}, *, y_{0}, y_{1}, \ldots\right\rangle\right) \neq x_{n}$ for any value of $*$. (Note that $*$ is irrelevant since player $n$ cannot see his own hat.)

For such a sequence, no player guesses correctly under the hat assignment $\left\langle x_{0}, x_{1}, \ldots\right\rangle$.

We begin with $X_{0}=\omega$. Suppose now that $n \geq 0$ and that we have constructed $X_{n}$ as well as $x_{k}$ for each $k$ with $0 \leq k<n$. Let $f:\left[X_{n}\right]^{\omega} \rightarrow \omega$ be given by $f(Y)=S_{n}\left(\left\langle x_{0}, \ldots, x_{n-1}, *, y_{0}, y_{1}, \ldots\right\rangle\right)$. By Lemma 2.1, we can choose $x_{n} \in X_{n}$ and $X_{n+1} \in\left[X_{n}\right]^{\omega}$ such that $x_{n}<\min \left(X_{n+1}\right)$ and for each $Y \in\left[X_{n+1}\right]^{\omega}, f(Y) \neq x_{n}$. That is, for each $Y \in\left[X_{n+1}\right]^{\omega}$, if $\left\langle y_{0}, y_{1}, \ldots\right\rangle$ is the increasing enumeration of $Y$, then $S_{n}\left(\left\langle x_{0}, \ldots, x_{n-1}, *, y_{0}, y_{1}, \ldots\right\rangle\right) \neq x_{n}$.

We now return to ZFC and consider the necessity of infinite visibility (or a cycle) for minimal predictors to exist. If two players can see each other, then there definitely exists a minimal predictor with two colors: one player guesses assuming the hats are the same color and the other guesses assuming they are not. This easily generalizes to show that minimal predictors exist with two colors whenever there is a cycle in the visibility graph [HT08a]. The following shows that for two colors, a cycle is the only way to get a minimal predictor with finite visibility.

THEOREM 2.3. With two colors and finite visibility on any set $P, a$ minimal predictor exists iff there exists a cycle in the visibility graph.

Proof. The case where $P$ is finite was shown in [HT08a]. A trivial consequence is that if there is a cycle, then there is a minimal predictor even if $P$ is infinite: just ignore all players and visibility outside the (finite) cycle. Another consequence is that, with acyclic visibility, for any predictor and 
any finite subset $F$ of players, there is a hat assignment under which everyone in $F$ guesses incorrectly: fix an arbitrary hat assignment for the players outside $F$; this induces a hat problem with finitely many players and acyclic visibility, and we can then make everyone in $F$ wrong by invoking the finite case.

Now, suppose we have finite acyclic visibility. We will show that no minimal predictor exists. Fix a predictor $S$. For $x \in P$, let $D_{x}=\left\{f \in{ }^{P_{2}}\right.$ : $S(f)(x) \neq f(x)\}$, the set of hat assignments under which $x$ guesses incorrectly, and let $\mathcal{D}=\left\{D_{x}: x \in P\right\}$. We must show that $\bigcap \mathcal{D} \neq \emptyset$. The above observation that we can make any finite set of players wrong shows that $\mathcal{D}$ has the finite intersection property. Put the usual product topology on ${ }^{P_{2}}$, which is compact by Tychonoff's theorem. Each $D_{x}$ is closed, since membership in $D_{x}$ depends only on the values of a function on the finite set $\{x\} \cup V(x)$. We have now shown that $\mathcal{D}$ is a family of closed subsets of a compact space, with the f.i.p., so $\bigcap \mathcal{D} \neq \emptyset$.

3. The transitive case in ZFC. The following result rather completely characterizes those transitive visibility relations for which a minimal predictor exists. The proof is essentially contained in [HT08b], but for the sake of completeness, we include it here.

TheOREM 3.1. For a transitive visibility relation $V$ on a set $P$, regardless of the number of colors, the following are equivalent:

(1) There exists an infinite sequence $\left\langle p_{i}: i \in \omega\right\rangle$ of distinct players such that $p_{n}$ sees $p_{n+1}$ for every $n \in \omega$.

(2) There exists an infinite set of players who have a finite-error predictor among themselves.

(3) There exists a minimal predictor.

Proof. Assume (1) holds. Then, because of transitivity, each of the countably many players in the sequence can see all but finitely many others in the sequence. Hence, by the argument for the Gabay-O'Connor theorem in the introduction, there is a finite-error predictor for this set of players, so (2) holds. Of course, $(2) \Rightarrow(3)$ is trivial. For the final implication, assume (1) fails and that $S$ is a predictor. We can define an increasing sequence $\left\langle B_{\alpha}: \alpha<\gamma\right\rangle$ of subsets of $P$ by letting $B_{\alpha}$ consist of those players who only see players in $\bigcup\left\{B_{\beta}: \beta<\alpha\right\}$. Notice that if $P \neq \bigcup\left\{B_{\beta}: \beta<\alpha\right\}$, then $B_{\alpha} \neq \emptyset$, since an infinite unsuccessful search for an element of $B_{\alpha}$ would yield an infinite sequence of players of the type we are assuming does not exist. With $\left\langle B_{\alpha}: \alpha<\gamma\right\rangle$ at hand, we can proceed by induction on $\alpha$ to define a hat assignment under which everyone in $P$ guesses wrong when using the predictor $S$. 
In $\mathrm{ZF}+\mathrm{DC}$, one can write a formula that expresses the existence of a minimal predictor, and this formula is independent of $\mathrm{ZF}+\mathrm{DC}$ for many visibility relations and sets of colors. In ZFC, the formula that expresses the existence of a minimal predictor in the transitive case is provably equivalent to the formula that expresses the existence of an infinite chain of visibility. However, that latter formula can still be independent of ZFC. For example, the question of whether a computable subtree of $\omega^{<\omega}$ is well-founded is $\Pi_{1}^{1}$-complete, so there are going to be computable trees for which their well-foundedness is independent of ZFC. These trees would induce transitive visibility relations (let a node see all its descendants) for which the existence of a predictor is independent of ZFC. So, the sense in which we understand the transitive case is more a matter of mathematical practice than any rigorous notion: our analysis of that case is complete in that we have carried the problem to the doorstep of well-foundedness and dropped it there.

4. The nontransitive case in $\mathrm{ZFC}$ and $\mathrm{ZFC}+\mathrm{CH}$. For some kinds of hat problems, the results in the transitive case carry over to the nontransitive case, but are just (apparently) harder to prove. For example, in the transitive case with two or more colors, a finite-error predictor exists iff there is no sequence $x_{n}$ of players such that $x_{i}$ cannot see $x_{j}$ for $i \leq j$, and the proof is fairly succinct; in the nontransitive case, the same holds when there are countably many players (the uncountable case is open), but the proof is more elaborate [Har10. So, it would be natural to speculate that the situation with minimal predictors is similar, and that Theorem 3.1 holds in the nontransitive case, but with a more complicated proof. However, it turns out that the nontransitive case is very unlike the transitive case here, and that minimal predictors can exist without having an infinite chain of visibility. More interestingly, the existence of minimal predictors can now depend on the number of colors, in some cases yielding independence results.

To exhibit these phenomena that distinguish the nontransitive case from the transitive case, we consider in this section and the next the visibility graph on $\omega$ in which evens see higher-numbered odds and odds see highernumbered evens. This directed graph is (essentially) isomorphic to the one on $P=2 \times \omega$ where $(i, m)$ sees $(j, n)$ iff $i \neq j$ and $m<n$. In what follows, we will freely switch back and forth between $\omega$ and $2 \times \omega$, and we will let EO denote the corresponding visibility relations.

The visibility relation EO is notable for having many infinite paths but being highly nontransitive in the sense that when $x \mathrm{EO} y$ and $y \mathrm{EO} z$ we never have $x \mathrm{EO} z$. We refer to the visibility relation $\mathrm{EO}$ as the even-odd context. 
Notationally, we will use $P^{0}$ for both the set of evens and $\{0\} \times \omega$, and $P^{1}$ for both the set of odds and $\{1\} \times \omega$. Similarly, we will often think of a hat assignment $f$ as a pair $\left(f^{0}, f^{1}\right)$ where $f^{i}$ specifies the hat assignment for $P^{i}$, and a predictor $S$ as a pair $\left(S^{0}, S^{1}\right)$ where $S^{i}:{ }^{\omega} \nu \rightarrow{ }^{\omega} \nu$ tells us how the players in $P^{i}$ guess (as a function of $f^{1-i}$ ).

The existence of minimal predictors in the even-odd context is quite dependent on the number of colors. The following result gives an outright positive result in $\mathrm{ZF}$, an outright negative result in $\mathrm{ZFC}$, and an extreme positive result under the additional assumption of $\mathrm{CH}$.

THEOREM 4.1.

(a) $\langle\omega, \mathrm{EO}\rangle \rightarrow\langle 1\rangle_{2}$.

(b) $\langle\omega, \mathrm{EO}\rangle \rightarrow\langle 1\rangle_{\omega_{2}}$.

(c) $\mathrm{CH} \vdash\langle\omega, \mathrm{EO}\rangle \rightarrow\langle 1\rangle_{\omega_{1}}$.

Proof. For (a), let players in $P^{0}$ guess 1 iff they see infinitely many players in $P^{1}$ with hat color 1 ; let players in $P^{1}$ guess 0 iff they see infinitely many players in $P^{0}$ with hat color 1 . If neither or both of $P^{0}$ and $P^{1}$ have infinitely many players with color 1 , then infinitely many players in $P^{0}$ will be correct; otherwise infinitely many players in $P^{1}$ will be correct.

For (b), fix any predictor $S=\left(S^{0}, S^{1}\right)$. For any ordinal $\alpha$, let $c_{\alpha}$ be the function on $\omega$ that is constantly $\alpha$. We intend to color $P^{0}$ with $c_{\beta}$ for some $\beta \in \omega_{1}$, and color $P^{1}$ with $c_{\gamma}$ for some $\gamma \in \omega_{2}$.

Since $\omega_{2}$ is regular, we can choose $\gamma \in \omega_{2}$ such that $\gamma>S_{n}^{1}\left(c_{\alpha}\right)$ for every $\alpha \in \omega_{1}$ and $n \in \omega$. Now choose $\beta \in \omega_{1}$ such that $\beta>S_{n}^{0}\left(c_{\gamma}\right)$ for every $n \in \omega$ such that $S_{n}^{0}\left(c_{\gamma}\right) \in \omega_{1}$. Then, under the hat assignment $\left(c_{\beta}, c_{\gamma}\right)$, everyone guesses incorrectly. (What is really going on in the above argument is that given functions $S^{0}: \omega_{2} \rightarrow \omega_{1}$ and $S^{1}: \omega_{1} \rightarrow \omega_{2}$, there is a pair $(\beta, \gamma)$ such that $\beta>S^{0}(\gamma)$ and $\gamma>S^{1}(\beta)$ : just choose $\gamma$ above the supremum of $S^{1}$, and $\beta$ above $S^{0}(\gamma)$.)

For (c), we only sketch the proof, as it will be a special case of more general results in the next section. Using $\mathrm{CH}$ we fix a well-ordering $\preceq$ of ${ }^{\omega} \omega_{1}$ of order type $\omega_{1}$. For any $f \in{ }^{\omega} \omega_{1}$, let $\hat{f}$ be $\preceq$-minimal such that $\hat{f}$ and $f$ eventually agree in the sense that there exists an $x \in \omega$ such that for all $y>x, \hat{f}(y)=f(y)$. Informally, the players' strategies will be that for a hat assignment $\left(f^{0}, f^{1}\right)$, the players in $P^{i}, i=0,1$, will assume $\hat{f}^{i} \preceq \hat{f}^{1-i}$ and guess according to a function that infinitely agrees with each $g \preceq \hat{f}^{1-i}$; at least one of those assumptions will turn out to be correct, yielding a minimal predictor.

Considering EO as a relation on $2 \times \omega$, one can form analogous relations on $\beta \times \gamma$ for other ordinals $\beta, \gamma$. Theorem 4.1 has extensions to these contexts; these will be discussed elsewhere. 
5. The nontransitive case in ZFC $+2^{\aleph_{0}}=\aleph_{2}$. Somewhat surprisingly, there seems to be a close connection between the existence of minimal predictors in the even-odd context and properties of the ideal of meager sets of real numbers. In fact, our consistency results make use of two prominent cardinal invariants; these and several others all lie between $\aleph_{1}$ and $2^{\aleph_{0}}$, and the relationships between them are well understood; a summary can be found in [Jec03, pp. 532-533] while [BJ95, Bar09, Mil81] provide detailed accounts. We say that functions $f, g \in{ }^{\omega} \nu$ infinitely agree if $f \cap g$ is infinite, and only finitely agree if $f \cap g$ is finite.

Definition 5.1. With $\mathcal{M}$ denoting the ideal of meager subsets of $\mathbb{R}$,

(a) $\operatorname{cov}(\mathcal{M})$ is the least cardinality of a subset of $\mathcal{M}$ whose union is $\mathbb{R}$;

(b) $\operatorname{non}(\mathcal{M})$ is the least cardinality of a nonmeager set.

LEMma 5.2 ([BJ95, pp. 54-59]).

(a) $\operatorname{cov}(\mathcal{M})$ is the smallest size of a family $F \subseteq{ }^{\omega} \omega$ such that $\left(\forall g \in{ }^{\omega} \omega\right)$ $(\exists f \in F) f$ and $g$ only finitely agree.

(b) $\operatorname{non}(\mathcal{M})$ is the smallest size of a family $F \subseteq{ }^{\omega} \omega$ such that $\left(\forall g \in{ }^{\omega} \omega\right)$ $(\exists f \in F) f$ and $g$ infinitely agree.

The following definition and lemma are key to the positive results in the even-odd context.

Definition 5.3. A family $G \subseteq{ }^{\omega} \nu$ is agreeable if for any $F \subseteq G$ with $|F|<|G|$, there is a $g \in G$ such that for each $f \in F, g$ and $f$ infinitely agree.

Lemma 5.4. If ${ }^{\omega} \nu$ is agreeable, then $\langle\omega, \mathrm{EO}\rangle \rightarrow\langle 1\rangle_{\nu}$.

Proof. Let $\lambda=\left|{ }^{\omega} \nu\right|$ and fix a well-ordering $\preceq$ of ${ }^{\omega} \nu$ of order type $\lambda$. For any $f \in \omega^{\omega} \nu$, let $\hat{f} \in{ }^{\omega} \nu$ be $\preceq$-minimal such that $\hat{f}$ and $f$ eventually agree. As in the proof of Theorem 4.1(c), the players' strategies will be that for a hat assignment $\left(f^{0}, f^{1}\right)$, the players in $P^{i}$ will assume $\hat{f}^{i} \preceq \hat{f}^{1-i}$ and guess according to a function that infinitely agrees with each $g \preceq \hat{f}^{1-i}$; at least one of those assumptions will turn out to be correct, yielding a minimal predictor.

We define $A:{ }^{\omega} \nu \rightarrow{ }^{\omega} \nu$ as follows. For any $f \in{ }^{\omega} \nu$, we have $\mid\left\{g \in{ }^{\omega} \nu\right.$ : $g \preceq \hat{f}\} \mid<\lambda$, so by the agreeability of ${ }^{\omega} \nu$, we can choose $A(f)$ to infinitely agree with each $g \preceq \hat{f}$. Note that for a hat assignment $\left(f^{0}, f^{1}\right)$, a player in $P^{i}$ can only see the values of $f^{1-i}$ on a tail of $\omega$, but this is enough information to determine $\hat{f}^{1-i}$ and hence $A\left(f^{1-i}\right)$. The predictor $S$ is defined by letting players in $P^{i}$ guess according to $A\left(f^{1-i}\right)$.

For any hat assignment $\left(f^{0}, f^{1}\right)$, we have $\hat{f}^{0} \preceq \hat{f}^{1}$ or $\hat{f}^{1} \preceq \hat{f}^{0}$. Suppose the former. Then $A\left(f^{1}\right)$ infinitely agrees with $\hat{f}^{0}$, and since $\hat{f}^{0}$ and $f^{0}$ even- 
tually agree, $A\left(f^{1}\right)$ also infinitely agrees with $f^{0}$, so infinitely many players in $P^{0}$ guess correctly. Similarly, if $\hat{f}^{1} \preceq \hat{f}^{0}$, infinitely many players in $P^{1}$ guess correctly.

An immediate consequence of Lemma 5.4 is the formal proof (promised in the last section) that $\mathrm{CH} \vdash\langle\omega, \mathrm{EO}\rangle \rightarrow\langle 1\rangle_{\omega_{1}}$ because ${ }^{\omega} \omega_{1}$ is agreeable, as the following argument shows. Note that under $\mathrm{CH},\left|{ }^{\omega} \omega_{1}\right|=\aleph_{1}$. Take any $F \subseteq{ }^{\omega} \omega_{1}$ with $|F|<\aleph_{1}$. Since $F$ is countable, we can produce a sequence of functions $f_{0}, f_{1}, \ldots \in{ }^{\omega} \omega_{1}$ in which each $f \in F$ appears infinitely often. The function $g(n)=f_{n}(n)$ infinitely agrees with every $f \in F$.

In fact, in models of ZFC $+\operatorname{non}(\mathcal{M})=2^{\aleph_{0}}=\aleph_{2},\langle\omega, \mathrm{EO}\rangle \rightarrow\langle 1\rangle_{\omega}$ is equivalent to a number of natural conditions, as the following theorem shows. This establishes the independence of $\langle\omega, \mathrm{EO}\rangle \rightarrow\langle 1\rangle_{\omega}$ from $\mathrm{ZFC}+\operatorname{non}(\mathcal{M})=2^{\aleph_{0}}=\aleph_{2}$ : adding $\aleph_{2}$ random reals to a model of $\mathrm{CH}$ yields a model in which $\operatorname{cov}(\mathcal{M})=\aleph_{1}+\operatorname{non}(\mathcal{M})=2^{\aleph_{0}}=\aleph_{2}$ [Mil81, p. 109], and it is well known that models of MA $+2^{\aleph_{0}}=\aleph_{2}$ have $\operatorname{cov}(\mathcal{M})=\operatorname{non}(\mathcal{M})=2^{\aleph_{0}}$ $=\aleph_{2}$.

Theorem 5.5. Assume $\operatorname{non}(\mathcal{M})=2^{\aleph_{0}}=\aleph_{2}$. Then the following are equivalent:

(1) $\operatorname{cov}(\mathcal{M})=\aleph_{2}$

(2) $\mathrm{MA}_{\aleph_{1}}$ (countable).

(3) ${ }^{\omega} \omega$ is agreeable.

(4) $\langle\omega, \mathrm{EO}\rangle \rightarrow\langle 1\rangle_{\omega}$.

Proof. (1) $\Leftrightarrow(2)$ can be found in [BJ95, p. 138].

$(1) \Rightarrow(3)$ is an easy consequence of Lemma 5.2 (a), but we offer a direct argument of $(2) \Rightarrow(3)$ for the sake of making the connection intuitively clear. Let $Q$ be the partial order of finite partial functions from $\omega$ to $\omega$, ordered by reverse inclusion, and note that $Q$ is countable. Take any $F \subseteq{ }^{\omega} \omega$ with $|F|<$ $\left|{ }^{\omega} \omega\right|=\aleph_{2}$. For $f \in F$ and $n \in \omega$, let $D_{f, n}=\{q \in Q: n \in \operatorname{dom}(q)$ \& $(\exists k \geq n) q(k)=f(k)\}$, which is dense in $Q$. Let $\mathcal{D}=\left\{D_{f, n}: f \in F, n \in \omega\right\}$. We have $|\mathcal{D}| \leq \aleph_{1}$, so by $\mathrm{MA}_{\aleph_{1}}$ (countable), there is a $\mathcal{D}$-generic filter $G \subseteq Q$. Letting $g=\bigcup Q \in{ }^{\omega} \omega, g$ infinitely agrees with each $f \in F$. Therefore, ${ }^{\omega} \omega$ is agreeable.

$(3) \Rightarrow(4)$ is immediate from Lemma 5.4 .

$(4) \Rightarrow(1)$. Supposing $\operatorname{cov}(\mathcal{M})=\aleph_{1}$, we will show $\langle\omega, \mathrm{EO}\rangle \rightarrow\langle 1\rangle_{\omega}$. Let $F$ be as in Lemma 5.2(a), with $|F|=\aleph_{1}$. Take any predictor $S=\left(S^{0}, S^{1}\right)$. We intend to color $P^{0}$ with some $f^{0} \in F$. Let $F^{\prime}=\left\{S^{1}(f): f \in F\right\}$. Since $\left|F^{\prime}\right| \leq \aleph_{1}<\operatorname{non}(\mathcal{M})$, Lemma $5.2(\mathrm{~b})$ gives us $f^{1} \in \omega_{\omega}$ such that $\left(\forall f \in F^{\prime}\right)$ $f$ and $f^{1}$ only finitely agree. By our choice of $F$, there exists $f^{0} \in F$ such that $f^{0}$ only finitely agrees with $S^{0}\left(f^{1}\right)$. Then $S$ has only finitely many 
correct guesses under hat assignment $f=\left(f^{0}, f^{1}\right)$, so $\langle\omega, \mathrm{EO}\rangle \nrightarrow\langle\omega\rangle_{\omega}$, and by Proposition 1.1 it follows that $\langle\omega, \mathrm{EO}\rangle \rightarrow\langle 1\rangle_{\omega}$.

6. Questions. We begin with a piece of terminology and an observation.

Definition 6.1. Say that $\left\langle f_{\alpha}: \alpha<\omega_{1}\right\rangle \subseteq{ }^{\omega} \nu$ is a strongly agreeable family in ${ }^{\omega} \nu$ if $\left(\forall f \in \omega^{\omega} \nu\right)\left(\exists \alpha \in \omega_{1}\right)(\forall \beta>\alpha) f$ and $f_{\beta}$ agree infinitely.

THEOREM 6.2. The following are equivalent:

(1) There exists a strongly agreeable family in ${ }^{\omega} \omega_{1}$.

(2) $\langle\omega, \mathrm{EO}\rangle \rightarrow\langle 1\rangle_{\omega_{1}}$.

Proof. (1) $\Rightarrow(2)$. Fix a strongly agreeable family $\left\langle f_{\alpha}: \alpha<\omega_{1}\right\rangle$ in ${ }^{\omega} \omega_{1}$. The predictor is as follows. For a hat assignment $h=\left(h^{0}, h^{1}\right)$, the players in $P^{i}$ choose $\alpha^{i}$ such that $\left(\forall \beta \geq \alpha^{i}\right) h^{1-i}$ and $f_{\beta}$ agree infinitely, and guess according to $f_{\alpha^{i}}$.

We must have $\alpha^{0} \leq \alpha^{1}$ or $\alpha^{1} \leq \alpha^{0}$. If $\alpha^{1-i} \leq \alpha^{i}$, then since $h^{i}$ agrees infinitely with $f_{\beta}$ for $\beta \geq \alpha^{1-i}, h^{i}$ agrees infinitely with $f_{\alpha^{i}}$, so infinitely many players in $P^{i}$ guess correctly.

$(2) \Rightarrow(1)$. Suppose there is no strongly agreeable family in $\omega_{\omega_{1}}$. Fix any predictor $S=\left(S^{0}, S^{1}\right)$. Let $c_{\alpha}$ be as in the proof of Theorem 4.1. Let $f_{\alpha}=$ $S^{1}\left(c_{\alpha}\right)$. Since $\left\langle f_{\alpha}: \alpha<\omega_{1}\right\rangle$ is not a strongly agreeable family, we can choose $g \in{ }^{\omega} \omega_{1}$ such that $\left(\forall \alpha \in \omega_{1}\right)(\exists \beta>\alpha) g$ only finitely agrees with $f_{\beta}$. In particular, there exists $\beta>\sup S^{0}(g)$ such that $g$ only finitely agrees with $f_{\beta}$. Then, under the hat assignment $\left(c_{\beta}, g\right)$, no player in $P^{0}$ guesses correctly since $\beta>\sup S^{0}(g)$, and only finitely many players in $P^{1}$ guess correctly since $g$ only finitely agrees with $f_{\beta}=S^{1}\left(c_{\beta}\right)$. This establishes $\langle\omega, \mathrm{EO}\rangle \nrightarrow\langle\omega\rangle_{\omega_{1}}$, so $\langle\omega, \mathrm{EO}\rangle \nrightarrow\langle 1\rangle_{\omega_{1}}$ by Proposition 1.1.

Theorem 6.2 might be of use in settling the following.

Question 6.3. Does $\langle\omega, \mathrm{EO}\rangle \rightarrow\langle 1\rangle_{\omega_{1}}$ imply $\mathrm{CH}$ ?

As we mentioned earlier, we are also unable to settle the following.

Question 6.4. Can one prove in ZFC that $\langle\omega, \mathrm{EO}\rangle \rightarrow\langle 1\rangle_{3}$ ?

We suspect, but have not proven, an affirmative answer to the following.

QuESTION 6.5. If $V$ is a visibility relation on a set $X$ with no infinite chain of visibility, must there exist $\kappa$ such that $\langle X, V\rangle \rightarrow\langle 1\rangle_{\kappa}$ ?

In our discussion of the necessity of some nontrivial version of the axiom of choice in producing minimal predictors on $\omega$ with full visibility, it was crucial that the number of colors was infinite. As we pointed out there, with finitely many colors, there are minimal predictors even if each player sees only the hats of higher-numbered players (thus avoiding the 
trivial solutions using cycles). The particular predictor we described had the players guess the least color they see occurring infinitely often. Even with two colors, this predictor is not "neutral" in the sense of respecting permutations of the set of colors; that is, a neutral predictor $S$ satisfies $S(\sigma \circ f)=\sigma \circ S(f)$ where $\sigma$ is any such permutation. This suggests the following.

Question 6.6. Can one prove in $\mathrm{ZF}+\mathrm{DC}$ that there exists a neutral minimal predictor when $P=\omega$ and each player sees only the hats of highernumbered players? If not, is the predictor described above essentially the only one that can be produced in $\mathrm{ZF}+\mathrm{DC}$ ?

Finally, how much visibility is needed for a minimal predictor to exist? Of course, this depends on the model, so we could ask two questions: how much visibility is needed for a minimal predictor to exist under $\mathrm{CH}$ ? How much is needed in the model where we have added $\aleph_{2}$ random reals?

\section{References}

[Bar09] T. Bartoszyński, Invariants of measure and category, in: Handbook of Set Theory, M. Foreman and A. Kanamori (eds.), Springer, Berlin, 2009, 491-555.

[BJ95] T. Bartoszyński and H. Judah, Set Theory: On the Structure of the Real Line, A K Peters, Wellesley, MA, 1995.

[Bla94] A. Blass, Cardinal characteristics and the product of countably many infinite cyclic groups, J. Algebra 169 (1994), 512-540.

[Bre95] J. Brendle, Evasion and prediction - the Specker phenomenon and Gross spaces, Forum Math. 7 (1995), 513-541.

[Bre03] -, Evasion and prediction III: Constant prediction and dominating reals, J. Math. Soc. Japan 55 (2003), 101-115.

[BS96] J. Brendle and S. Shelah, Evasion and prediction II, J. London Math. Soc. 53 (1996), 19-27.

[BS03] -, Evasion and prediction IV: Strong forms of constant prediction, Arch. Math. Logic 42 (2003), 349-360.

[Gal65] F. Galvin, Problem 5348, Amer. Math. Monthly 72 (1965), 1136.

[GP76] F. Galvin and K. Prikry, Infinitary Jonsson algebras and partition relations, Algebra Universalis 6 (1976), 367-376.

[Har10] C. S. Hardin, On transitive subrelations of binary relations, preprint, Union Coll., 2010.

[HT08a] C. S. Hardin and A. D. Taylor, An introduction to infinite hat problems, Math. Intelligencer 30 (2008), no. 4, 20-25.

[HT08b] - A peculiar connection between the axiom of choice and predicting the future, Amer. Math. Monthly 115 (2008), 91-96.

[HT09] -, Limit-like predictability for discontinuous functions, Proc. Amer. Math. Soc. 137 (2009), 3123-3128.

[Jec03] T. J. Jech, Set Theory. The Third Millenium Edition, Revised and Expanded, Springer Monogr. Math., Springer, Berlin, 2003.

[Mat77] A. R. D. Mathias, Happy families, Ann. Math. Logic 12 (1977), 59-111. 
[Mil81] A. W. Miller, Some properties of measure and category, Trans. Amer. Math. Soc. 266 (1981), 93-114; Corrections and additions, ibid. 271 (1982), 347-348.

Christopher S. Hardin, Alan D. Taylor

Department of Mathematics

Union College

Schenectady, NY 12308, U.S.A.

E-mail: hardinc@union.edu taylora@union.edu

Received 6 December 2009;

in revised form 12 February 2010 